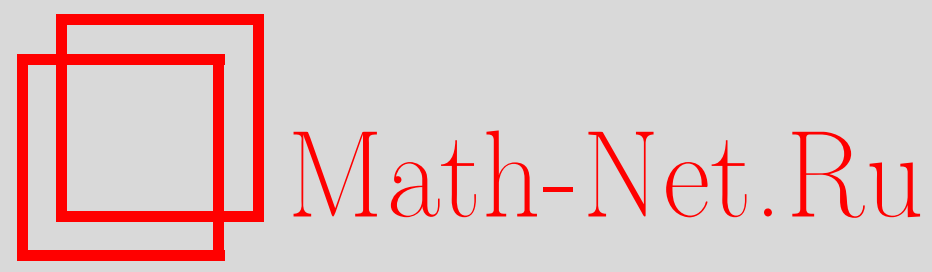

Р. И. Подловченко, В. Е. Хачатрян, Минимальность и тупиковость многоленточных автоматов, Дискрет. матем., 2008, том 20, выпуск 2, 100-121

DOI: https://doi.org/10.4213/dm1006

Использование Общероссийского математического портала Math-Net.Ru подразумевает, что вы прочитали и согласны с пользовательским соглашением http://www . mathnet.ru/rus/agreement

Параметры загрузки:

IP : 52.6 .47 .48

26 апреля 2023 г., $13: 22: 32$ 


\title{
Минимальность и тупиковость многоленточных автоматов
}

\author{
() 2008 г. Р. И. Подловченко, В. Е. Хачатрян
}

\begin{abstract}
Рассматривается класс $M$ детерминированных бинарных двухленточных автоматов, для которых состояния автомата, в которых происходит считывание с добавочной второй ленты, позволяют продолжить вычисления только в том случае, когда считываемый символ является фиксированным для всех автоматов. Для автоматов из класса $M$ решается так называемая обобщенная проблема минимизации. Эта проблема состоит в том, чтобы для всякого класса эквивалентных автоматов из $M$ найти в этом классе все минимальные по числу состояний автоматы. В статье доказана разрешимость обобщенной проблемы минимизации в $M$, описана и проанализирована процедура ее решения.
\end{abstract}

Работа выполнена при поддержке Российского фонда фундаментальных исследований, проект 06-01-00106.

\section{1. Введение}

Многоленточные автоматы, как самостоятельная модель вычислений, введены в [1] в качестве естественного обобщения обычных конечных автоматов, состоящего в переходе от одной ленты, несущей информацию для работы автомата, к нескольким лентам. В [1] установлено принципиальное отличие функциональных свойств многоленточных автоматов от одноленточных, а именно, неразрешимость проблемы включения для многоленточных автоматов и тот факт, что выразительная мощность недетерминированных многоленточных автоматов превышает таковую для детерминированных многоленточных автоматов.

Следующим важным результатом, подтверждающим отличие многоленточных автоматов от конечных, является установленная в [2] неразрешимость проблемы эквивалентности для недетерминированных многоленточных автоматов. Напомним, что проблема эквивалентности заключается в поиске алгоритма, который, получив на свой вход два автомата из рассматриваемого множества, устанавливает, эквивалентны они или нет.

Специально отметим, что обнаруженные свойства многоленточных автоматов проявляются уже в случае, когда лент две.

Подавляющая часть дальнейших исследований многоленточных автоматов посвящена проблеме эквивалентности в случае их детерминированности. Не останавливаясь на всех полученных здесь результатах, отметим те, что являются в известном смысле заключительными. В [3] показана разрешимость проблемы эквивалентности для двухленточных автоматов, а в [4] разрешимость в случае любого количества лент. 
Мы обратились к изучению практически незатронутой исследованиями характеристике многоленточных автоматов - минимальности автомата по его размерам, то есть по числу состояний. Исходя из опыта предшествующих исследований, мы ограничились рассмотрением детерминированных двухленточных автоматов, выделив среди них бинарные, то есть работающие над двухбуквенным алфавитом. Последнее опирается на то, что согласно [5] эквивалентность автоматов над произвольным (не менее, чем двухбуквенным) алфавитом сводится к эквивалентности именно бинарных автоматов.

Нами установлено следующее принципиальное отличие двухленточных автоматов (следовательно, и многоленточных) от обычных конечных: существуют классы эквивалентных детерминированных бинарных автоматов, содержащие несколько минимальных по размеру автоматов. Это нарушает известную картину для конечных автоматов, где минимальным в классе эквивалентных автоматов является всегда единственный автомат, им является тупиковый автомат, то есть такой, который не имеет двух различных эквивалентных состояний. В случае же двухленточных автоматов минимальные тоже являются тупиковыми, но, как мы показали, тупиковых автоматов может быть и бесконечное число в одном и том же классе эквивалентных автоматов.

Эти результаты получены для детерминированных бинарных двухленточных автоматов, структура которых незначительно отличается от структуры конечных, то есть одноленточных автоматов, а именно, состояния автомата, в которых происходит считывание с добавочной второй ленты, позволяют продолжить завершаемое вычисление только в случае, когда считываемый символ является заранее фиксированным для всех автоматов. Рассматриваемое множество автоматов обозначено $M$.

Цель наших исследований заключается в том, чтобы описать процедуру, посредством которой для всякого класса эквивалентных автоматов из $M$ выявляются все минимальные в этом классе автоматы. Их всегда конечное число. Поставленная задача именуется обобщенной проблемой минимизации в $M$.

Основным результатом данной статьи является выбранный нами метод решения обобщенной проблемы минимизации. Мы отказались от использования алгоритма, распознающего эквивалентность детерминированных бинарных автоматов, обратившись к структурному их анализу. Последний заключается в применении лишь эквивалентных преобразований автоматов при поиске минимальных автоматов. Для этого предварительно конструируется система $T$ эквивалентных преобразований, полная в $M$, то есть позволяющая конечным числом преобразований, принадлежащих $T$, трансформировать любой автомат из $M$ в эквивалентный ему.

Статья состоит из введения и пяти разделов. В разделе 2 определяются детерминированные бинарные двухленточные автоматы общего вида, после чего описывается рассматриваемое нами множество $M$. Здесь же приводятся примеры автоматов из $M$, подтверждающие свойства, наличие которых обосновывает нетривиальность обобщенной проблемы минимизации в $M$. Раздел 3 посвящен построению системы $T$, а раздел 4 - доказательству ее полноты в $M$ (теорема 1), а также сводимости проблемы обобщенной минимизации в $M$ к одноименной проблеме в подмножестве $\bar{M}$ множества $M$ (теорема 2). При доказательстве теоремы 1 в произвольном классе эквивалентности, содержащемся в $M$, строится канонический в нем автомат, который оказывается единственным в этом классе. Канонический автомат является тупиковым, но в общем случае не минимальным, что подтверждается примером. Демонстрируется возможность уменьшить размер канонического автомата, не теряя при этом свойства тупиковости. Это используется при доказательстве теоремы 2. В разделах 5 и 6 исследуются автоматы из множества $\bar{M}$. Каждый из них содержит как $p$-состояния, в которых осуществляется считывание с основной ленты, так и $q$-состояния, в которых происходит считывание с добавочной ленты. Раздел 5 
открывается тем, что произвольный класс эквивалентности $K$, содержащийся в $\bar{M}$, разбивается на подклассы, называемые срезами класса $K$. Инвариантом среза является одно и то же число $p$-состояний в принадлежащих срезу автоматах. Срез, содержащий канонический в $K$ автомат, характеризуется наименьшим значением инварианта и называется главным. Далее в разделе 5 излагается стратегия поиска минимальных в $K$ автоматов. Она предписывает, во-первых, исследование главного среза, нацеленное на построение в нем минимальных автоматов, и во-вторых, поиск в неглавных срезах автоматов, размеры которых не превышают размера минимальных автоматов в главном срезе, с доказательством их тупиковости и конечности их множества.

В разделе 5 описываются преобразования автоматов, используемые при реализации этой стратегии. Раздел 6 посвящен собственно реализации этой стратегии.

В заключение мы приводим теорему 3, утверждающая разрешимость обобщенной проблемы минимизации в множестве $M$.

Отметим, что полученные нами результаты не имеют аналогов в литературе по многоленточным автоматам. Сколько-нибудь близкой по тематике исследований является работа [6], но в ней обсуждается поиск хотя бы одного минимального автомата и в случае, когда рассматриваемое множество автоматов отлично от $M$.

Настоящей статье предшествовала статья [7], в которой дано решение обобщенной проблемы минимизации в $M$ в ситуации, когда допустимые срезы класса $K$ исчерпываются главным срезом; последнее выявляется эффективно.

\section{2. Множество $M$ детерминированных бинарных двухленточных автоматов и их свойства}

Как было указано во введении, начнем с определения детерминированного бинарного двухленточного автомата общего вида, позаимствовав его из [5].

Полагаем, что используемый нами алфавит $\Sigma$, над которым строятся такие автоматы, состоит из символов 0 и 1 , и впредь детерминированный бинарный двухленточный автомат, краткости ради, будем называть просто автоматом.

Структура автомата описывается следующим образом. Задается конечное множество $V$, элементы которого называются состояниями автомата. В $V$ выделяются три различных состояния: инициальное $v_{0}$, финальное $v^{*}$ и мертвое $\bar{v}$. Задается функция переходов автомата из одного состояния в другое под воздействием поступившего символа из $\Sigma$; она имеет вид $\left.\left(V \backslash\left\{v^{*}, \bar{v}\right)\right\}\right) \times \Sigma \rightarrow V$. Имеются две бесконечные ленты, заполненные символами из $\Sigma$ и называемые $p$-лентой и $q$-лентой. Каждая лента снабжается считывающей головкой, которая в любой момент времени обозревает какой-либо один символ своей ленты и способна сдвигаться вправо на одну позицию после считывания обозреваемого символа. Всякому состоянию из $V \backslash\left\{v^{*}, \bar{v}\right\}$ сопоставляется либо символ $p$, либо символ $q$; в первом случае состояние называется $p$-состоянием, во втором $-q$-состоянием.

Функционирование автомата представляет собой пошаговое его выполнение на заданной паре лент. На первом шаге автомат находится в состоянии $v_{0}$, и каждая из считывающих головок обозревает первый символ на своей ленте. Предположим, что в общем случае автомат находится в состоянии $v$, и каждая из считывающих головок обозревает некоторый символ на своей ленте. Если $v$ отлично от $v^{*}$ и $\bar{v}$, то происходит выполнение очередного шага. Оно совершается по следующему сценарию. Если $v$ - это $p$-состояние, то считывается обозреваемый символ с $p$-ленты, если $v$ - это $q$-состояние, то с $q$-ленты. После этого сработавшая головка сдвигается на своей ленте на одну позицию вправо, 
а состояние $v$ переходит в состояние, определяемое функцией переходов по считанному символу. В случае, когда $v=v^{*}$, автомат останавливается, и результатом его выполнения на заданной паре лент объявляется пара слов в алфавите $\Sigma$ : первое из них - это считанное с $p$-ленты, второе - считанное с $q$-ленты. Если же $v=\bar{v}$, то автомат останавливается тоже, но его выполнение на заданной паре лент квалифицируется как безрезультатное. Безрезультатным считается и его выполнение, если оно бесконечно.

Два автомата над $\Sigma$, по определению, эквивалентны, если, какой бы ни была заданная пара лент, либо оба останавливаются на ней с общим результатом, либо выполнение обоих на этой паре лент является безрезультатным.

Переведем данное определение автомата на используемый далее графический язык. Элементы множества $V$ будем представлять вершинами конечного ориентированного графа, которые по-прежнему будем называть состояниями, различая $p$-состояния и $q$-состояния посредством меток вершин символами $p$ и $q$ соответственно. Из любого состояния $v$, отличного от $v^{*}$ и $\bar{v}$, выведем две дуги, помеченные символами 0 и 1 соответственно и ведущие в состояния, определяемые функцией переходов.

Каждому ориентированному пути $w$ в этом графе сопоставим два слова в алфавите $\Sigma$, называемые $p$-проекцией и $q$-проекцией пути $w$. Первая составлена последовательным выписыванием символов, которыми помечены дуги, исходящие из $p$-состояний пути $w$; дуги просматриваются без пропусков и в порядке их следования в $w$; если $w$ не содержит $p$-состояний, то $p$-проекция пути $w$ - пустое слово. Аналогично определяется $q$-проекция пути $w$, но теперь вместо $p$-состояний рассматриваются $q$-состояния.

Два пути в графе назовем эквивалентными, если совпадают их $p$-проекции и отдельно $q$-проекции.

Если путь $w$ ведет из состояния $v_{0}$ в состояние $v^{*}$, то он называется путем через автомат.

Легко увидеть, что два автомата, представленные описанными выше графами, эквивалентны тогда и только тогда, когда, каким бы ни был путь через один из них, существует эквивалентный ему путь через другой.

Традиционным образом определяется отношение эквивалентности состояний, принадлежащих одному или различным автоматам. Автомат, не имеющий двух различных и эквивалентных состояний, называется тупиковым. Если последний имеет наименьшее число состояний среди всех эквивалентных ему автоматов, то он называется минимальным в своем классе эквивалентности.

Теперь от общих понятий перейдем к определению множества $M$. Оно состоит из всех автоматов, структура которых удовлетворяет следующим требованиям: из любого $q$-состояния автомата дуга, помеченная символом 1, ведет в мертвое состояние автомата; любое состояние автомата, отличное от мертвого, принадлежит какому-либо пути через автомат.

Функционирование автомата из $M$, как нетрудно видеть, характеризуется свойством: если автомат останавливается на какой-либо паре лент, то он останавливается с тем же результатом и в случае, когда $q$-лента заменена на такую, которая заполнена только символом 0. Это свойство послужило основанием тому, что в [7] автоматы из $M$ названы автоматами, работающими с лентами, одна из которых всегда заполнена символом 0 . Неточность названия компенсируется его образностью.

Обратимся к примерам, демонстрирующим свойства автоматов из $M$, послужившие основанием к рассмотрению обобщенной проблемы минимизации в $M$ (ее формулировка дана во введении).

Условимся при изображении автоматов пользоваться следующими соглашениями. Состояния автомата маркируются символами $p$ и $q$, будучи лишены своих обозначений. 


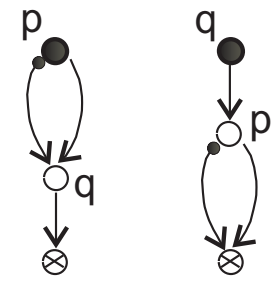

Pис. 1.
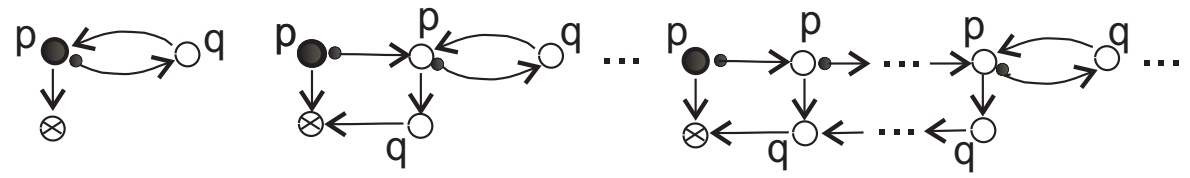

Pис. 2.

Дуга, помеченная символом 1 и исходящая из $p$-состояния, снабжается жирной точкой у ее основания, что позволяет не выставлять метки дуг. Мертвое состояние автомата вместе с приходящими в него дугами не изображается, инициальное состояние изображается затемненным кружочком, финальное - кружочком с крестиком.

На рис. 1 представлены два автомата из $M$, эквивалентность и минимальность которых очевидна.

На рис. 2 изображена бесконечная серия эквивалентных автоматов, каждый из которых является тупиковым, что нетрудно установить.

\section{3. Система $T$ эквивалентных преобразований автоматов из $M$}

Сначала изложим концепции, которым подчиняется построение систем эквивалентных преобразований (э. п.) автоматов. Они описаны в [8] для моделей вычислений, объекты которых - это размеченные конечные графы. Мы спроецируем эти концепции на рассматриваемый нами случай, ограничиваясь содержательным описанием.

Согласно [8], системы э. п. создаются в лоне исчисления фрагментов.

Фрагмент автомата - это его часть, порожденная некоторым множеством состояний автомата и всеми инцидентными им дугами. Состояния и инцидентные им дуги наследуют метки, присвоенные им в автомате. Эти состояния считаются принадлежащими фрагменту.

Связь фрагмента с остальной частью автомата задается множеством приходящих в него дуг из состояний, не принадлежащих фрагменту, и исходящих из него дуг, ведущих в состояния, ему не принадлежащие. Начало и конец каждой приходящей дуги назовем внешним и соответственно внутренним входами фрагмента, а исходящей - внутренним и внешним выходами фрагмента.

Учитывая структуру автоматов, принадлежащих множеству $M$, введем понятие допустимого фрагмента. Таковым будем считать фрагмент, удовлетворяющий требованиям:

(1) мертвое и финальное состояния могут быть только внешними выходами фрагмента; 
(2) если фрагмент не имеет приходящих в него дуг, то он содержит инициальное состояние;

(3) всякое состояние, принадлежащее фрагменту, находится на ориентированном пути, ведущем к его внешнему выходу либо из внутреннего входа, либо из принадлежащего фрагменту инициального состояния.

Отметим, что отсюда вытекают следующие свойства допустимого фрагмента: из всякого его внутреннего входа имеется ориентированный путь, ведущий к внешнему выходу фрагмента; если фрагменту принадлежит инициальное состояние, то из него ориентированным путем достижим какой-либо внешний выход; существуют внешние выходы, отличные от мертвого состояния.

Пусть $F_{1}, F_{2}$ - допустимые фрагменты, обладающие следующими свойствами: множество состояний одного из них является подмножеством множества состояний другого, возможно, совпадающим с последним; оба множества одновременно либо содержат инициальное состояние, либо не содержат его.

Говорим, что фрагменты $F_{1}, F_{2}$ согласованы, если между приходящими в них дугами установлено взаимно однозначное соответствие, сохраняющее метки дуг, а также установлено взаимно однозначное соответствие между внешними выходами фрагментов, и при этом выполнены следующие условия: совпадают начала соответствующих друг другу приходящих дуг и совпадают соответствующие друг другу внешние выходы.

Для согласованных фрагментов $F_{1}, F_{2}$ определяется операция замены в автомате вхождения в него фрагмента $F_{i}, i=1,2$, вхождением фрагмента $F_{3-i}$, состоящая в подстановке вместо приходящих дуг и внешних выходов фрагмента $F_{i}$ соответствующих им дуг и внешних выходов другого.

Операция замены трансформирует автомат из $M$ в автомат из $M$.

Два согласованных фрагмента называются эквивалентными, если какими бы ни были автомат и вхождение в него одного из согласованных фрагментов, операция замены его другим фрагментом является эквивалентным преобразованием.

Каждая пара эквивалентных фрагментов индуцирует множество э. п. автоматов.

Система э. п. автоматов задается множеством, состоящим из пар эквивалентных фрагментов. Последнее разбито на подмножества, называемые аксиомами. Традиционное требование к аксиоме состоит в том, чтобы она была разрешимым множеством и, кроме того, вместе с любой принадлежащей ей парой фрагментов содержала и ее обращение.

Опишем множества $B 1$ и $B 2$ пар допустимых фрагментов автоматов из $M$.

Множество $B 1$ состоит из пар фрагментов $\left(F_{1}, F_{2}\right)$ и $\left(F_{2}, F_{1}\right)$, удовлетворяющих следующим требованиям. Фрагмент $F_{1}$ допускает разбиение множества всех его состояний на непересекающиеся классы $V_{1}, \ldots, V_{k}$ такие, что

(1) все состояния одного класса - это либо $p$-состояния, либо $q$-состояния;

(2) если некоторая дуга, выходящая из состояния класса $V_{i}, i=1, \ldots, k$, ведет в состояние класса $V_{j}, j=1, \ldots, k$, то одинаково с ней помеченная дуга, выходящая из любого состояния класса $V_{i}$, тоже ведет в состояние класса $V_{j}$;

(3) если некоторая дуга, выходящая из состояния класса $V_{i}, i=1, \ldots, k$, является исходящей для фрагмента $F_{1}$, то одинаково с ней помеченные дуги, выходящие из других состояний класса $V_{i}$, тоже являются исходящими дугами фрагмента $F_{1}$, и все они ведут в одно и то же состояние. 

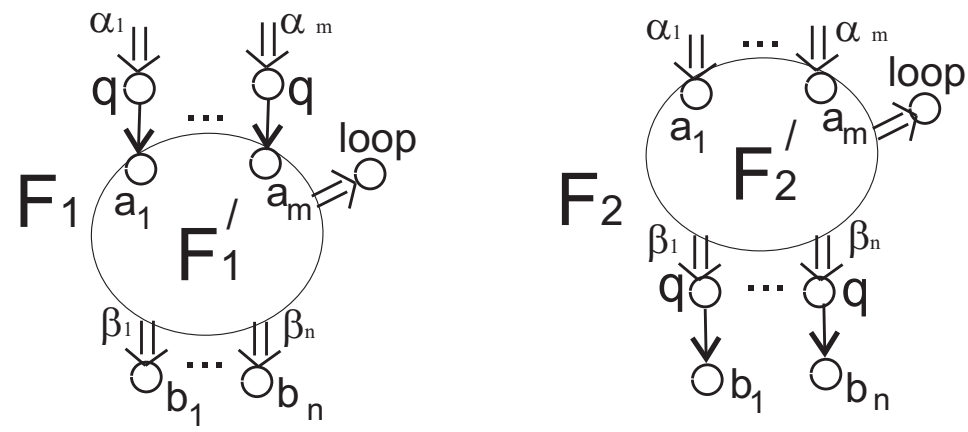

Pис. 3.

Фрагмент $F_{2}$ получается из $F_{1}$ следующим построением. В каждом классе $V_{i}$, $i=1, \ldots, k$, выделяется одно состояние $v_{i}$, совпадающее с инициальным состоянием автомата, если последнее принадлежит классу $V_{i}$, и выбранное произвольно в остальных случаях. Если выходящая из $v_{i}$ дуга вела в состояние класса $V_{j}, i=1, \ldots, k$, то она направляется в состояние $v_{j}$. Всякая приходящая дуга фрагмента $F_{1}$, оканчивающаяся в каком-либо состоянии класса $V_{i}$, направляется в состояние $v_{i}$. Состояния, отличающиеся от выделенных, опускаются.

Согласование фрагментов $F_{1}, F_{2}$ осуществляется естественным образом.

Если пара $\left(F_{1}, F_{2}\right)$ принадлежит множеству $B 1$, то очевидной является эквивалентность $F_{1}, F_{2}$.

Отсюда вытекает справедливость следующего утверждения.

Предложение 1. Множество В1-аксиома.

Преобразование, индуцируемое парой $\left(F_{1}, F_{2}\right)$ из $B 1$ и заключающееся в замене фрагмента $F_{1}$ фрагментом $F_{2}$, назовем склейкой состояний и обозначим $B 1 \downarrow$, а в замене $F_{2}$ фрагментом $F_{1}$ расклейкой состояний и обозначим $B 1 \uparrow$.

Охарактеризуем дополнительно преобразование по аксиоме $B 1$. В этих целях введем отношение сильной эквивалентности автоматов из $M$. Рассмотрим преобразование $\psi$ произвольного автомата $A$ из $M$ в обычный конечный автомат над алфавитом $\{p, q\} \times\{0,1\}$, состоящее в следующем: всякая дуга $t$ автомата $A$ трансформируется в дугу, помеченную парой $(s, \varepsilon)$, где $s-$ метка состояния, из которого выходит дуга $t$, а $\varepsilon-$ метка самой дуги $t$; полагаем далее, что в конструируемом автомате все переходы из мертвого состояния ведут в него же и туда ведут все переходы из финального состояния.

По определению, автоматы $A_{1}, A_{2}$ из $M$ сильно эквивалентны тогда и только тогда, когда эквивалентны конечные автоматы $\psi\left(A_{1}\right), \psi\left(A_{2}\right)$. Очевидно, что из сильной эквивалентности следует обычная.

Легко доказать следующее утверждение.

Предложение 2. Всякое преобразование по аксиоме В1 является сильно эквивалентным.

Перейдем к описанию множества $B 2$. Полагаем, что оно состоит из всех пар допустимых фрагментов $\left(F_{1}, F_{2}\right)$ и $\left(F_{2}, F_{1}\right)$, где вид $F_{1}, F_{2}$ представлен на рис. 3. Прокомментируем его. Двойные стрелки на рисунке обозначают множество дуг, приходящих в состояние, где оканчивается стрелка. Совпадение меток, приписанных этим стрелкам, 
означает наличие взаимно однозначного соответствия между дугами помеченных множеств, сохраняющего как метки самих дуг, так и состояния, из которых исходят эти дуги. Мертвое состояние обозначено "lоор".

Через $F_{1}^{\prime}$ и $F_{2}^{\prime}$ обозначены непустые подфрагменты фрагментов $F_{1}, F_{2}$, имеющие общими принадлежащие им состояния и отличающиеся входящими в них дугами и внешними выходами, как это показано на рис. 3. Полагаем, что фрагменты $F_{1}^{\prime}$ и $F_{2}^{\prime}$ содержат p-состояния.

Состояния $a_{1}, \ldots, a_{m}$, где $m \geqslant 1$, принадлежат каждому из фрагментов $F_{1}^{\prime}, F_{2}^{\prime}$, а состояния $b_{1}, \ldots, b_{n}, n \geqslant 1$, являются внешними выходами фрагментов $F_{1}$ и $F_{2}$.

Возможен случай, когда одно из множеств $\alpha_{1}, \ldots, \alpha_{m}$ пусто; если это $\alpha_{i}$, то $q$-состояние, в которое направлена двойная стрелка, помеченная $\alpha_{i}$, является инициальным, и тогда во фрагменте $F_{2}^{\prime}$ инициальным является состояние $a_{i}$.

Нетрудно доказать следующее утверждение.

Предложение 3. Преобразования, индуцируемые парами из В2, являются эквивалентньми, то есть В2 - аксиома.

Если $\left(F_{1}, F_{2}\right) \in B 2$, то замену фрагмента $F_{1}$ фрагментом $F_{2}$ будем записывать как $B 2 \downarrow$, а замену $F_{2}$ фрагментом $F_{1}-$ как $B 2 \uparrow$.

Отметим, что в отличие от $B 1$ преобразования по аксиоме $B 2$ не являются сильно эквивалентными.

Обозначим $T$ систему э. п. автоматов, индуцируемую аксиомами $B 1$ и $B 2$.

\section{4. Доказательство полноты системы $T$ в $M$ и сведение обобщенной проблемы минимизации в $M$ к этой проблеме в $\bar{M}$}

Полнота системы $T$ устанавливается следующим традиционным путем. Доказывается, что в каждом классе эквивалентности в $M$ имеется единственный его представитель; он называется каноническим автоматом и конструируется применением конечной цепочки преобразований из $T$.

Отсюда следует, что какими бы ни были два эквивалентных автомата из $M$, построив цепочки преобразований, приводящие их к канонической форме, и обратив одну из них, мы получим конечную цепочку, трансформирующую средствами системы $T$ один из взятых автоматов в другой.

Построению канонического автомата предшествует построение по заданному автомату эквивалентного ему так называемого q-упорядоченного автомата; последний конструируется конечной цепочкой преобразований из $T$.

Введем используемые понятия.

Фрагменты $F_{1}$ и $F_{2}$ с рис. 3 будем называть соответственно фрагментом с нижней границей и фрагментом с верхней границей.

Пусть $F^{\prime}$ и $F^{\prime \prime}-$ фрагменты с нижней границей. Назовем их обособленными, если у них отсутствуют общие принадлежащие им состояния и общие внешние выходы. Говорим, что $F^{\prime}$ вложен во фрагмент $F^{\prime \prime}$, если первый является подфрагментом второго.

Пусть $F-$ фрагмент с нижней границей. Строгим вхождением $F$ в автомат назовем вхождение, обладающее следующим свойством: к множеству состояний, принадлежащих фрагменту $F$, нельзя добавить новых состояний, не нарушая при этом его статуса быть фрагментом с нижней границей. 


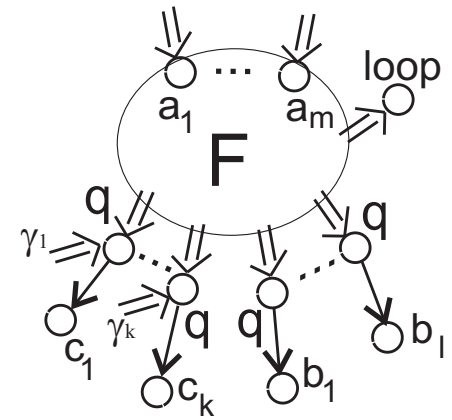

Рис. 4.
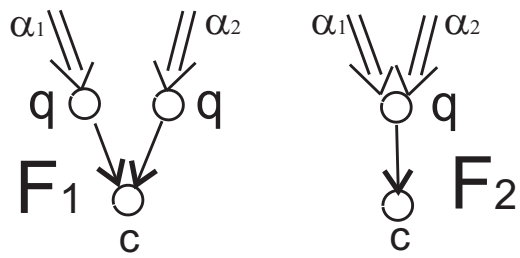

Рис. 5.

Важное свойство строгого вхождения фиксируется предложением 4.

Предложение 4. Если $F$ - строго входящиий в автомат фрагмент с нижней границей, то преобразованием В2个 он трансформируется во фрагмент $F^{\prime}$ с верхней границей, которая не является нижней границей какого-либо фрагмента преобразованного автомата. При этом, если $F$ вложен в какой-либо фрагмент $F^{\prime \prime}$ исходного автомата, то и $F^{\prime}$ в преобразованном автомате остается вложенным в модифицированный фрагмент $F^{\prime \prime}$.

Обратимся к рис. 4. Изображенный на нем фрагмент назовем $t$-фрагментом, где $t \geqslant 0$. Здесь, как и раньше, множества $\gamma_{1}, \ldots, \gamma_{k}$ не пусты и состоят из дуг, которые ведут в состояния, где оканчиваются двойные стрелки с метками этих множеств. Если $t=0$, то $t$-фрагмент - это фрагмент с нижней границей.

Введем аксиому $B 0$, являющуюся подмножеством аксиомы $B 1$. По определению, $B 0$ состоит из всех пар фрагментов $\left(F_{1}, F_{2}\right)$ и $\left(F_{2}, F_{1}\right)$, где $F_{1}, F_{2}$ изображены на рис. 5. Здесь $\alpha_{1}, \alpha_{2}-$ непустые множества дуг, приходящих в изображенные на рис. $5 q$-состояния.

Отметим, что применением аксиомы $B 0$ всякий $t$-фрагмент трансформируется в фрагмент с нижней границей.

Опишем теперь процедуру $\delta$, которая применяется к автомату $A$ из $M$ и строит автомат $\delta(A)$, принадлежащий $M$.

На первом этапе работы процедуры $\delta$ автомат $A$ применением аксиомы $B 0$ преобразуется в эквивалентный ему автомат $A^{\prime}$, обладающий следующим свойством: в любое $q$-состояние, принадлежащее автомату $A^{\prime}$, входит в точности одна дуга.

Очевидно, что в автомате $A^{\prime}$ отсутствуют вхождения $t$-фрагментов с $t=0$. Обозначим $Q$ множество строго входящих в $A^{\prime}$ фрагментов с нижней границей. Понятно, что $Q-$ конечное множество и любые два фрагмента из $Q$ либо являются обособленными, либо один из них вложен в другой. 
Если $Q$ пусто, то полагаем, что $\delta(A)=A^{\prime}$. Если же $Q$ не пусто, то, просматривая принадлежащие $Q$ фрагменты в некотором порядке, процедура $\delta(A)$ к каждому из них применяет преобразование $B 2 \downarrow$. Тот факт, что при этом не появятся новые фрагменты с нижней границей, следует из предложения 4.

Из описания процедуры $\delta$ следует, что если $Q$ пусто, то $\delta(A)-$ единственный построенный автомат, если же $Q$ не пусто, то при любом его упорядочивании процедура $\delta$ строит один и тот же (с точностью до изоморфизма) автомат $\delta(A)$. Автомат $\delta(A)$ назовем $q$-упорядоченным. Очевидно, что в $\delta(A)$ нет $t$-фрагментов.

Предложение 5. Прочедура $\delta(A)$ реализуется конечной изепочкой преобразований из $T$.

Действительно, выделение в автомате строгих вхождений фрагментов с нижней границей осуществляется без применения его преобразований, а все трансформации, используемые алгоритмом $\delta$, - это преобразования по аксиомам $B 0$ и $B 2$.

Докажем следующее утверждение.

Лемма 1. В q-упорядоченном автомате из $M$ из эквивалентности его состояний следует сильная их эквивалентность.

Доказательство. Пусть $A-q$-упорядоченный автомат из $M$, а $a$ и $b-$ эквивалентные состояния автомата $A$. Если $a, b$ не являются сильно эквивалентными, то в $A$ существуют эквивалентные состояния $a^{\prime}, b^{\prime}$ такие, что $a^{\prime}$ - это $p$-состояние, а $b^{\prime}$ - это $q$-состояние. В силу эквивалентности $a^{\prime}, b^{\prime}$ на каждом пути из $a^{\prime}$ в финальное состояние автомата $A$ обязательно встретится $q$-состояние. Построим в $A$ фрагмент, состоящий из $p$-состояний, лежащих на путях из $a^{\prime}$ к первому встречающемуся $q$-состоянию, и из тех $q$-состояний из числа последних, для которых непосредственные их преемники принадлежат пройденным $p$-состояниям. Этот фрагмент обозначим $F$.

В общем случае $F$ является $t$-фрагментом с $t>0$. Действительно, из допустимости фрагмента $F$ следует существование хотя бы одного его внешнего выхода, являющегося $q$-состоянием.

Таким образом, наличие фрагмента $F$ противоречит $q$-упорядоченности автомата $A$. Лемма 1 доказана.

Следствие 1. Любые два эквивалентных q-упорядоченных автомата из $M$ являются сильно эквивалентными.

Рассмотрим теперь класс $K$ эквивалентных автоматов из $M$. Для каждого из них существует единственный построенный по нему $q$-упорядоченный автомат, и все эти автоматы сильно эквивалентны. Выполнением $B 1 \downarrow$-преобразований, склеивающих сильно эквивалентные состояния, каждый $q$-упорядоченный автомат трансформируется в единственный в классе $K$ автомат, не имеющий различных и сильно эквивалентных состояний. Этот автомат назовем каноническим в $K$. Очевидно, что он тупиковый.

Предложение 6. Существует алгоритм, который по любому автомату из $M$ строит канонический автомат в классе эквивалентности взятого автомата.

Наконец, докажем следующую теорему.

Теорема 1. Система $T$ полна в $M$.

Доказательство. Для любой пары $A_{1}, A_{2}$ эквивалентных автоматов из $M$ нужно установить существование конечной цепочки преобразований, принадлежащих $T$, трансформирующую $A_{1}$ в $A_{2}$. Эта цепочка выстраивается из двух цепочек, каждая из которых по 

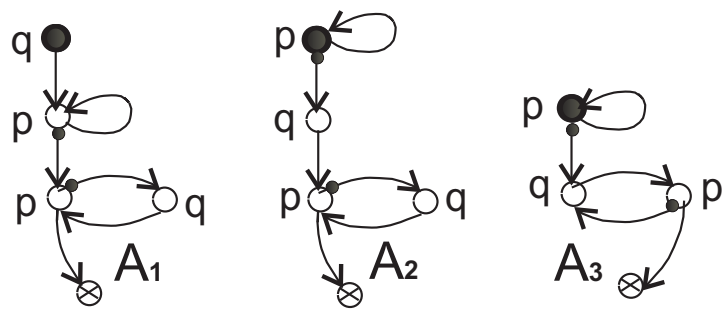

Рис. 6.

автомату сначала конструирует эквивалентный ему $q$-упорядоченный автомат (см. предложение 5), а затем трансформирует его в канонический, применяя в конечном числе преобразования $B 1 \downarrow$. Обратив одну из этих двух цепочек, мы получим требуемую. Теорема доказана.

Отметим, что из предложения 6 следует разрешимость в $M$ проблемы эквивалентности.

Покажем теперь на примере, что число $q$-состояний в каноническом автомате можно уменьшить, не лишаясь при этом свойства тупиковости.

На рис. 6 представлены автоматы $A_{1}, A_{2}, A_{3}$. Легко проверить, что $A_{1}-$ канонический автомат, ибо он $q$-упорядочен и не имеет сильно эквивалентных состояний.

Автомат $A_{2}$ получается применением $B 2 \downarrow$-преобразования к автомату $A_{1}$. В автомате $A_{2}$ его $q$-состояния сильно эквивалентны, поэтому к нему применимо $B 1 \downarrow$-преобразование, склеивающее эти два $q$-состояния и трансформирующее $A_{2}$ в автомат $A_{3}$. Последний автомат тупиковый и меньший по размеру, чем автомат $A_{1}$.

Анализ только что разобранной ситуации состоит в следующем. Автомат $A_{1}$, в сущности, является цепочкой из трех автоматов, в которой финальное состояние предшествующего автомата является инициальным для последующего. Автоматы, входящие в цепочку, по типу классифицируются на автоматы одиночные $q$-состояния, автоматы, содержащие только $p$-состояния, и автоматы смешанного типа, то есть содержащие как $p$-состояния, так и $q$-состояния. Входящий в $A_{1}$ автомат смешанного типа является частным случаем автомата, в котором инициальным является $p$-состояние и существует ориентированный путь из него в $q$-состояние, преемником которого является это $p$-состояние; назовем в этом автомате данное $q$-состояние выделенным, а сам автомат особым. Легко убедиться в справедливости следующего утверждения.

Предложение 7. В ичепочке, состоящеей из одиночного q-состояния и примыкающего к нему особого автомата, сильно эквивалентными являются это одиночное q-состояние и выделенное q-состояние особого автомата. Если же к одиночному q-состоянию примыкает автомат, отличный от особого, то в такой цепочке нет различных и сильно эквивалентных q-состояний.

Обозначим $\bar{M}$ подмножество множества $M$, состоящее их всех автоматов смешанного типа и докажем следующую теорему.

Теорема 2. Обобщенная проблема минимизации в $М$ сводится к одноименной проблеме в $\bar{M}$.

Доказательство. Любой канонический автомат из $M$ представим в виде максимальной цепочки его подавтоматов. Если в ней присутствуют особый автомат и одиночное 


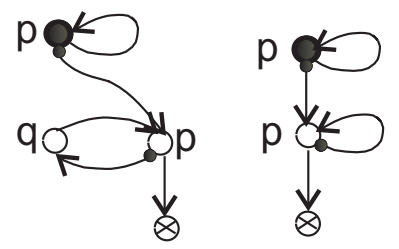

Pис. 7.

$q$-состояние, то преобразованием по аксиоме $B 2$ перенесем одиночное $q$-состояние к особому автомату, после чего по аксиоме $B 1$ склеим появившиеся сильно эквивалентные $q$-состояния. Проделаем эту операцию, пока она возможна. Согласно предложению 7 в модифицированной цепочке каждый содержащийся в ней автомат является каноническим в своем классе эквивалентности. Очевидно, что одиночные $q$-состояния и автоматы из $p$-состояний уже минимальны каждый в своем классе эквивалентности. Таким образом, задача построения минимальных в классе эквивалентности, которому принадлежит исходный канонический автомат, сводится к поиску минимальных в классах эквивалентности, представленных автоматами смешанного типа. Теорема 2 доказана.

\section{5. Срезы класса эквивалентности в $\bar{M}$ и $\varphi$-преобразование}

Введем понятие среза класса эквивалентности в $\bar{M}$ и опишем инструмент, используемый при исследовании срезов, так называемое $\varphi$-преобразование. Далее устанавливаются факты, относящиеся к области применения $\varphi$-преобразований.

Для произвольного автомата $A$ из $\bar{M}$ введем понятие его $p$-проекции. Это, по определению, автомат, содержащий только $p$-состояния и построенный применением к $A$ следующей процедуры. Она состоит из двух этапов. Первый этап заключается в обработке циклов в автомате $A$ и опускается при отсутствии их. Если же циклы имеются, то каждый из них обрабатывается следующим образом: рассматриваются приходящие в цикл дуги, конец каждой такой дуги совмещается с ближайшим $p$-состоянием, расположенном в цикле на пути, начинающемся этой дугой. На втором этапе процедуры в трансформированном (в общем случае) автомате рассматриваются максимальные линейные отрезки, внутренние вершины которых не имеют извне входящих в них дуг. В каждом из таких отрезков опускаются дуги, идущие из q-состояний, без нарушения целостности отрезка. При этом, если какая-либо из опускаемых дуг начинается в инициальном состоянии, то инициальным объявляется ближайшее к ней $p$-состояние, находящееся в отрезке.

По окончании второго этапа построен автомат из $p$-состояний.

Легко проверяемым является следующее утверждение.

Предложение 8. Любая детерминизация описанной процеедуры приводит к одному и тому же результату.

Дадим примеры построения $p$-проекции. На рис. 7 слева изображен автомат, полученный из автомата $A_{3}$ с рис. 6 выполнением первого этапа процедуры; справа приводится результат выполнения второго этапа процедуры, то есть $p$-проекция автомата $A_{3}$. Легко увидеть, что при построении $p$-проекции автомата $A_{1}$ с того же рис. 6 первый этап отсутствует, а второй приводит к автомату, изображенному справа на рис. 7. 


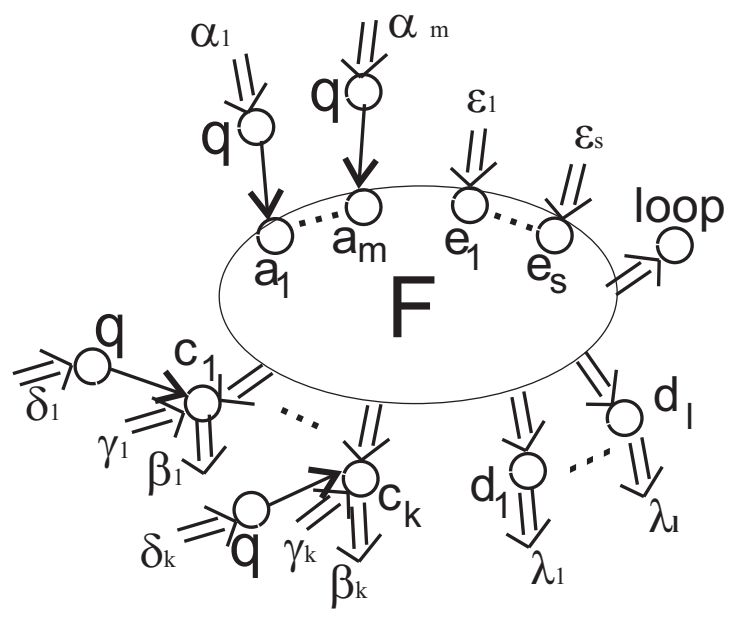

Рис. 8.

Рассмотрим какой-либо класс эквивалентности $K$, принадлежащий множеству $\bar{M}$. Разобьем $K$ на подклассы, называемые его срезами, полагая, что срез состоит из всех автоматов из $K$, имеющих одну и ту же $p$-проекцию.

Обозначим $T_{0}$ систему э. п., индуцируемую аксиомами $B 0$ и $B 2$.

Справедливо следующее утверждение.

Предложение 9. Система $T_{0}$ полна в любом срезе класса $K$.

Действительно, преобразованиями по аксиомам $B 0$ и $B 2$ любой автомат из среза трансформируется в $q$-упорядоченный, а он единствен в срезе.

Вполне очевидно, что минимальным автоматом в отдельном срезе класса $K$ является тупиковый автомат, размеры которого не могут быть уменьшены преобразованиями из $T_{0}$, и что минимальным автоматом в самом классе $K$ является наименьший по размеру из всех минимальных автоматов в его срезах.

Во введении описана стратегия поиска минимальных автоматов в классе эквивалентности из $\bar{M}$.

Она применяется к автоматам из $\bar{M}$, обладающим следующим свойством: ни одно состояние автомата не является преемником двух и более $q$-состояний. Множество таких автоматов обозначим $\widetilde{M}$.

Рабочим инструментом при осуществлении стратегии является так называемое $\varphi$-преобразование. Опишем фрагмент автомата из $\widetilde{M}$, к которому оно применяется. Он изображен на рис. 8. Прокомментируем этот рисунок. Здесь $a_{1}, \ldots, a_{m}, m \geqslant 1$, и $e_{1}, \ldots, e_{s}$, $s \geqslant 0$, являются внутренними входами фрагмента $F$, при этом $a_{1}, \ldots, a_{m}-$ преемники $q$-состояний и только их, являющихся внешними входами фрагмента $F$, а $e_{1}, \ldots, e_{s}-$ это $q$-состояния, и каждое из них является преемником хотя бы одного $p$-состояния, не принадлежащего фрагменту $F$.

Внешние выходы $c_{1}, \ldots, c_{k}, k \geqslant 0$, фрагмента $F$ являются преемниками $q$-состояний, не принадлежащих фрагменту $F$, а внешние выходы $d_{1}, \ldots, d_{l}, l \geqslant 0$, не обладают этим свойством, то есть если и являются преемниками состояний, не принадлежащих $F$, то только $p$-состояний. Предполагается, что $k+l>0$. 
Двойные стрелки, изображенные на рис. 8 и либо ведущие в loор, либо обозначенные символами $\gamma_{1}, \ldots, \gamma_{k}$, могут представлять собой и пустые множества дуг, все остальные двойные стрелки представляют непустые множества дуг.

Фрагмент с рис. 8 назовем $s$-фрагментом. Очевидно, что при $s=0$ это обычный фрагмент с верхней границей.

Условимся входы $a_{1}, \ldots, a_{m}$ и выходы $d_{1}, \ldots, d_{l}$ называть активными, а входы $e_{1}, \ldots, e_{s}$ и выходы $c_{1}, \ldots, c_{k}$ пассивными. Пассивные входы, если они имеются, разобьем на две группы, отнеся к первой те из них, преемниками которых являются состояния из фрагмента $F$, а ко второй те, преемниками которых являются внешние выходы фрагмента $F$. Отметим сразу, что это активные внешние выходы, ибо пассивный выход (в силу принадлежности множеству $\widetilde{M}$ автомата, которому принадлежит рассматриваемый $s$-фрагмент) не может служить преемником $q$-состояния из $F$. Понятно, что любая из групп может быть пустой.

Обозначим $s$-фрагмент, изображенный на рис. 8 , через $\widetilde{F}$. Как уже было отмечено, фрагмент $\widetilde{F}$ поступает на вход $\varphi$-преобразования, которое описывается ниже и результатом которого будет $t$-фрагмент автомата из $\widetilde{M}$.

В общем случае $\varphi$-преобразование состоит из трех этапов. Первый этап выполняется только при $s>0$. Его цель - получить из фрагмента $\widetilde{F}$ обычный фрагмент с верхней границей. Это осуществляется так. К каждому пассивному входу фрагмента $\widetilde{F}$ применяется аксиома $B 0$, посредством которой от него отклеивается одна копия. Если вход принадлежит первой группе, то отклеенная копия становится внешним входом фрагмента $F$, если же она принадлежит второй группе, то ее преемником становится тот внешний выход, который был преемником рассматриваемого пассивного входа.

Очевидно, что цель этапа достигается. Обозначим $\widetilde{F}^{\prime}$ фрагмент, в который трансформировался фрагмент $\widetilde{F}$. Полагаем, что при отсутствии первого этапа $\widetilde{F}^{\prime}=\widetilde{F}$.

На втором этапе к фрагменту $\widetilde{F}^{\prime}$ применяется $B 2 \downarrow$-преобразование, трансформирующее его в $t$-фрагмент. Последний в общем случае не обладает свойствами фрагмента автомата из $\widetilde{M}$.

Действительно, если $k>0$, то всякий пассивный выход стал преемником двух $q$-состояний. Аналогичное свойство приобрел и тот активный выход, в который вела дуга из пассивного входа, если, конечно, были пассивные входы второй группы.

Цель третьего этапа - вернуться в множество $\widetilde{M}$. Это осуществляется склеиванием появившихся пар $q$-состояний, нарушающих принадлежность полученного $t$-фрагмента автомату из $\widetilde{M}$. Склеивание обеспечивает аксиома $B 0$.

Итак, $\varphi$-преобразование завершено. Фрагмент $\varphi(\underset{F}{\tilde{F}})$ изображен на рис.9. Здесь $e_{1}^{\prime}, \ldots, e_{r}^{\prime}-$ преемники пассивных входов фрагмента $\widetilde{F}$, принадлежащих первой группе.

На рис. 10 продемонстрировано применение $\varphi$-преобразования. Здесь представлены автоматы $A_{1}, A_{2}, A_{3}$. Очевидно, что автомат $A_{1}$ принадлежит множеству $\widetilde{M}$. В $A_{1}$ штриховым прямоугольником выделен $s$-фрагмент, поступающий на вход $\varphi$-преобразования; в этом фрагменте $m=2, s=1, k=0, l=1$. Автомат $A_{2}$ получен выполнением первого этапа $\varphi$-преобразования, а автомат $A_{3}$ - выполнением второго этапа. В $A_{2}$ и $A_{3}$ штриховыми прямоугольниками охвачены фрагменты, в которые последовательно трансформируется $s$-фрагмент, выделенный в $A_{1}$. В нем $k=0$ и отсутствуют пассивные входы второй группы, поэтому выполнение $\varphi$-преобразования завершается вторым этапом.

Поскольку в дальнейшем $\varphi$-преобразования будут применяться для уменьшения числа $q$-состояний в автомате или для сохранения их числа, рассмотрим существенные с этой позиции параметры $s$-фрагмента. Нетрудно видеть, что параметры $k$ и $s$ не влияют на число $q$-состояний, остающихся в автомате по завершении $\varphi$-преобразования, имен- 


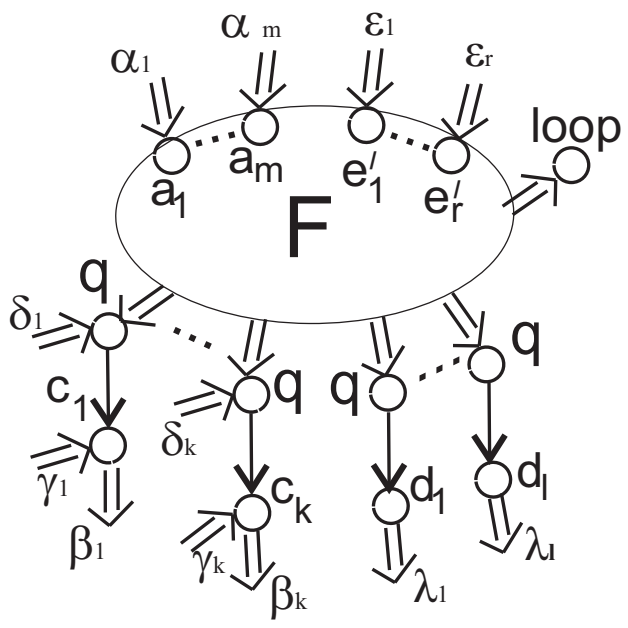

Рис. 9.
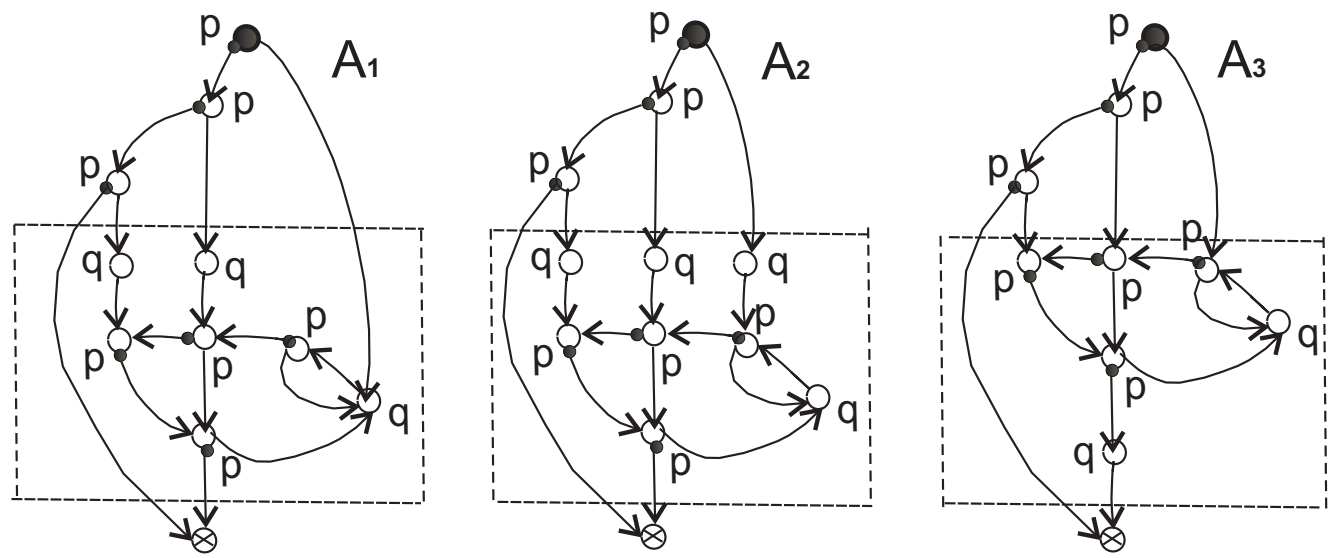

Рис. 10.

но поэтому входы $c_{1}, \ldots, c_{k}$ и выходы $e_{1}, \ldots, e_{s}$ названы пассивными. Таким образом, существенными параметрами $s$-фрагмента являются $m$ и $l$.

Легко видеть, что $\varphi$-преобразование при $m>l$ уменьшает число $q$-состояний в автомате, при $m<l$ увеличивает это число, а при $m=l$ оставляет тем же. В связи с этим разность $m-l$ назовем маркером $s$-фрагмента, а $\varphi$-преобразование $s$-фрагмента назовем успешным, если его маркер положителен, и устойчивым, если он равен 0.

Рис. 10 дает пример успешного $\varphi$-преобразования.

Отметим теперь используемые в дальнейшем факты и понятия.

Из самого описания $\varphi$-преобразования следует приводимое ниже утверждение. 


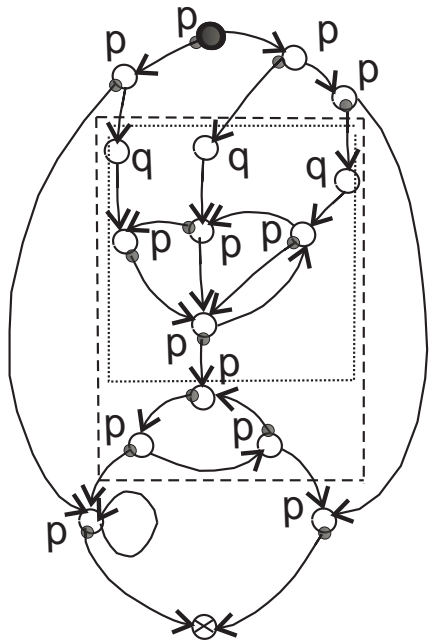

Рис. 11.

Предложение 10. Автомат не выводится $\varphi$-преобразованием из среза, которому он принадлежит.

Говорим, что $s$-фрагмент $F_{1}$ вложен в $s$-фрагмент $F_{2}$, если первый является подфрагментом второго, и выполнено требование, состоящее в том, что внешние входы фрагмента $F_{1}$ являются состояниями фрагмента $F_{2}$. Далее полагаем, что $t$-фрагмент $F_{1}$ вложен в $s$-фрагмент $F_{2}$, если первый является подфрагментом второго, и его выходы - это состояния фрагмента $F_{2}$.

Легко убедиться в справедливости следующего утверждения.

Предложение 11. Если $F_{1}, F_{2}-s$-фрагменты автомата из $\widetilde{M}$, и фрагмент $F_{1}$ вложен в $F_{2}$, то фрагмент $\varphi\left(F_{1}\right)$ вложен во фрагмент, полученный этим $\varphi$-преобразованием из фрагмента $F_{2}$.

Вхождение $s$-фрагмента в автомат из $\widetilde{M}$ назовем строгим, если к множеству принадлежащих ему состояний нельзя добавить новых, не нарушая при этом его статуса быть $s$-фрагментом.

На рис. 11 представлен автомат, в котором строго входящий в него $s$-фрагмент с двумя активными выходами имеет своим подфрагментом уже не строго входящий $s$-фрагмент с теми же входами, что и первый, и с одним выходом. Оба фрагмента заключены в штриховые прямоугольники, один из которых вложен в другой. Маркеры обоих фрагментов положительны. Очевидно, что для уменьшения числа $q$-состояний в автомате с рис. 11 следует применить $\varphi$-преобразование к вложенному $s$-фрагменту, маркер которого больше маркера объемлющего его $s$-фрагмента.

Этот пример приводит к введению ряда понятий для $s$-фрагмента, строго входящего в автомат $\widetilde{M}$. 
Всякий подфрагмент фрагмента $F$, имеющий своими входами входы фрагмента $F$, назовем его префиксом. Если единственным префиксом фрагмента $F$ является он сам, то припишем фрагменту $F$ нулевой ранг; в противном случае говорим, что $F$ имеет ненулевой ранг.

Характеристикой $F$ назовем максимальный маркер его префиксов, а префиксы $F$, имеющие максимальный маркер, называем представителями $F$.

Справедливо следующее утверждение.

Предложение 12. Пусть $F^{\prime}-$ один из представителей фрагмента $F$. Нижняя граница фрагмента $\varphi\left(F^{\prime}\right)$ в случае, когда $F^{\prime}=F$, не является верхней границей какого-либо s-фрагмента преобразованного автомата; если же $F^{\prime} \neq F$, то она является таковой.

Первая часть предложения 12 основана на том, что $F$ - строго входящий в автомат $s$-фрагмент; вторая - на том, что $F^{\prime}$ отличен от $F$.

Во втором случае $s$-фрагмент, возникающий в результате $\varphi$-преобразования фрагмента $F^{\prime}$, назовем индуцируемым им.

Справедливо следующее утверждение.

Предложение 13. Если $F$ не является представителем самого себя, то среди его представителей присутствует такой, для которого индуцируемый им s-фрагмент имеет маркер, меньший характеристики $F$.

Действительно, маркер индуцируемого фрагмента не может быть больше маркера обсуждаемого представителя. А в случае, когда он ему равен, к множеству представителей $F$ можно добавить еще один.

Представитель фрагмента $F$, существование которого устанавливается предложением 13 , назовем главным для $F$. К главным отнесем и $F$, если он является представителем самого себя.

\section{6. Построение минимальных автоматов в главном срезе}

Пусть $K$ - рассматриваемый нами класс эквивалентности в $\bar{M}$. Главным называется срез, которому принадлежит канонический в $K$ автомат. Поскольку этот автомат может быть эффективно построен, поиски минимальных в $K$ автоматов начнем с отыскания минимальных автоматов в главном срезе. Отметим, что при этом на основании предложения 9 мы располагаем только преобразованиями из системы $T_{0}$.

Из очевидной минимальности $p$-проекции канонического автомата вытекает следующее утверждение.

Предложение 14. Автоматьл из главного среза класса $K$ имеют минимальную р-проекциию среди всех автоматов из $K$.

Из построения канонического в $K$ автомата вытекает следующее утверждение. 

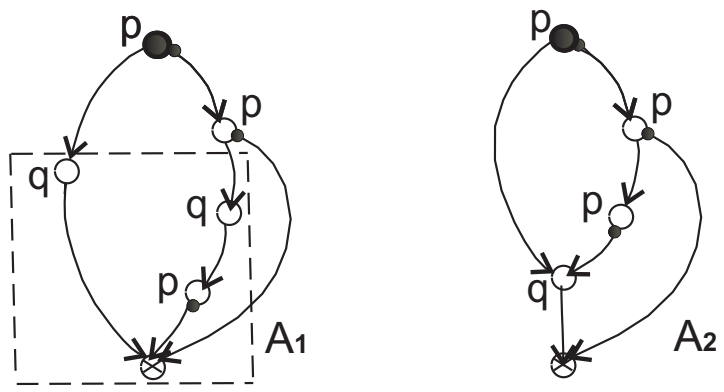

Рис. 12.

Предложение 15. В каноническом автомате отсутствуют $t$-фрагментыл.

Доказательство. Действительно, их нет в $q$-упорядоченном автомате из $K$, а его трансформация в канонический осуществляется склеиванием сильно эквивалентных состояний, при котором $p$-состояния склеиваются с $p$-состояниями, а $q$-состояния - с $q$-состояниями. Понятно, что при этом не могут появиться $t$-фрагменты.

Специально отметим, что канонический автомат принадлежит множеству $\widetilde{M}$. Тот факт, что в нем могут быть $s$-фрагменты, подтверждается примером канонического автомата $A_{1}$ на рис. 12. Принадлежащий ему $s$-фрагмент заключен в штриховой прямоугольник и является обычным фрагментом с верхней границей.

На этом основании будем рассматривать $\varphi$-преобразования канонического автомата, имеющего вхождения $s$-фрагментов, и докажем лемму 2.

Лемма 2. Любая конечная цеепочка $\varphi$-преобразований трансформирует канонический 8 К автомат в тупиковый.

Доказательство. Доказательство леммы проводится индукцией по числу выполненных $\varphi$-преобразований в предположении, что таковые имеются.

Опишем базис индукции. Пусть $A_{0}-$ канонический автомат в $K, F_{0}-s$-фрагмент в $A_{0}, F_{0}^{\prime}=\varphi\left(F_{0}\right)$ и $A_{0}^{\prime}$ - автомат, в который выполнением этого $\varphi$-преобразования трансформировался автомат $A_{0}$.

Чтобы доказать тупиковость автомата $A_{0}^{\prime}$, разобьем его состояния на подмножества $C_{0}, C_{1}, C_{2}$, где в $C_{0}$ включим состояния, образующие нижнюю границу фрагмента $F_{0}^{\prime}$, в $C_{1}$ включим состояния из $F_{0}^{\prime}$, не принадлежащие $C_{0}$, а в $C_{2}-$ все остальные состояния автомата $A_{0}^{\prime}$.

Состояния, принадлежащие $C_{2}$, попарно неэквивалентны, так как они наследуют это свойство из автомата $A_{0}$. Состояния из $C_{1}$ тоже попарно неэквивалентны, ибо они подверглись одинаковому воздействию выходящих из них путей, а в $A_{0}$ были попарно неэквивалентны.

Состояния из $C_{0}$ обладают следующим свойством: никакие два из них не имеют общего преемника. Вследствие того, что их преемники, находясь в $C_{2}$, попарно неэквивалентны, состояния из $C_{0}$ также попарно неэквивалентны.

Остается показать попарную неэквивалентность состояний, принадлежащих соответственно либо $C_{0}$ и $C_{1}$, либо $C_{0}$ и $C_{2}$, либо $C_{1}$ и $C_{2}$. Все три случая разберем, исходя из предположения, что имеются эквивалентные состояния, и приходя к противоречию. Всякий раз предполагаемые эквивалентными состояния обозначим $a$ и $b$. 
Случай 1. Пусть $a \in C_{0}, b \in C_{1}$. Если $b$ - это $q$-состояние, то $a^{\prime}$ и $b^{\prime}$, являющиеся преемниками состояний $a, b$ соответственно, эквивалентны. Состояние $a^{\prime}$ принадлежит $C_{2}$, а состояние $b^{\prime}-$ либо $C_{0}$, либо $C_{1}$. Оба случая рассматриваются дальше.

Пусть $b$ - это $p$-состояние. Тогда в автомате $A_{0}$ состояние $b$ эквивалентно состоянию $a^{\prime}$, и так как $b \in C_{1}, a^{\prime} \in C_{2}$, их эквивалентность противоречит тупиковости автомата $A_{0}$, ибо они не равны. Случай 1 исследован полностью.

Случай 2. Пусть $a \in C_{0}, b \in C_{2}$. Сначала рассмотрим ситуацию, когда $b-$ это $q$-состояние. Как и в предыдущем случае, преемники $a^{\prime}, b^{\prime}$ состояний $a$ и $b$ должны быть эквивалентными. Состояние $a^{\prime}$ принадлежит $C_{2}$, а состояние $b^{\prime}-$ либо $C_{0}$, либо $C_{1}$, либо $C_{2}$.

Вариант, когда $b^{\prime} \in C_{0}$, имеет место, если $s$-фрагмент $F_{0}$ обладал пассивным выходом $c_{i}$ (см. рис. 8 и 9) и состояние $b$ принадлежало множеству $\delta_{i}$. В автомате $A_{0}$ преемник состояния $b$ (он отличен от $b^{\prime}$, ибо в результате $\varphi$-преобразования исчез), как нетрудно заметить, эквивалентен состоянию $a^{\prime}$; оба они принадлежат $C_{2}$ и заведомо различны, то есть получено желаемое противоречие с тупиковостью автомата $A_{0}$.

Если $b^{\prime} \in C_{1}$, то этот случай рассматривается дальше.

Пусть $b^{\prime} \in C_{2}$. Равенство $a^{\prime}=b^{\prime}$ влечет за собой равенство $a=b$, так как в автомате $A_{0}^{\prime}$ не может быть $q$-состояний с общим преемником. Равенство $a$ и $b$ противоречит нашему предположению.

Разберем теперь ситуацию, когда $b$ - это $p$-состояние. Тогда в $A_{0}^{\prime}$ существует $t$-фрагмент, содержащий $b$ и поэтому отличный от фрагмента $F_{0}^{\prime}$. Это противоречит тому, что согласно предложению 15 , фрагмент $F_{0}^{\prime}$ - единственный $t$-фрагмент в $A_{0}^{\prime}$. Случай 2 полностью разобран.

Случай 3. Пусть $a \in C_{1}, b \in C_{2}$. Рассмотрим простые эквивалентные пути, ведущие из $a$ и $b$ в финальное состояние автомата $A_{0}^{\prime}$. Утверждаем, что среди пар состояний, принадлежащих этим путям и равноудаленных от их начала, имеется пара разнотипных состояний. Действительно, предполагая противное, мы придем к равенству $a=b$, ибо в $A_{0}^{\prime}$ нет различных и эквивалентных $p$-состояний и отсутствуют $q$-состояния с общим преемником.

Пусть $\bar{a}, \bar{b}-$ первая встретившаяся пара разнотипных состояний. Очевидно, что $\bar{a}$ и $\bar{b}$ эквивалентны. Предположим, что $\bar{a}-$ это $p$-состояние, то есть $\bar{b}-$ это $q$-состояние. Тогда $\bar{a}$ принадлежит либо $C_{1}$, либо $C_{2}$, а $\bar{b}-$ либо $C_{0}$, либо $C_{1}$, либо $C_{2}$. Рассмотрим все шесть вариантов.

Вариант 1: $\bar{a} \in C_{1}, \bar{b} \in C_{0}$. В этом случае в автомате $A_{0}$ эквивалентными являются состояние $\bar{a}$ и преемник состояния $\bar{b}$; обозначим его $\bar{b}^{\prime}$. Состояние $\bar{b}^{\prime}$ принадлежит либо $C_{1}$, либо $C_{2}$. Первая ситуация имеет место, если $\bar{b}$ во фрагменте $F_{0}$ принадлежит его верхней границе. Тогда заведомо исключается равенство $a=b^{\prime}$, и так как $\bar{a}$ и $\bar{b}^{\prime}$ находятся в $C_{1}$, мы приходим к противоречию с тупиковостью автомата $A_{0}$. Если же $\bar{b}^{\prime} \in C_{2}$, то это опять противоречит тупиковости автомата $A_{0}$.

Вариант 2 , где $\bar{a} \in C_{1}, \bar{b} \in C_{1}$, исключается сразу ввиду того, что $\bar{a} \neq \bar{b}$, а множество $C_{1}$ не имеет различных и эквивалентных принадлежащих ему состояний.

Bариант 3: $\bar{a} \in C_{1}, \bar{b} \in C_{2}$. Тогда, как и в варианте 1 , в автомате $A_{0}$ эквивалентными являются состояние $\bar{a}$ и преемник состояния $\bar{b}$, который обозначим $\bar{b}^{\prime}$. Поскольку $\bar{b}$ не принадлежит $C_{0}$, его преемник $\bar{b}^{\prime}$ находится в $C_{2}$. Но в автомате $A_{0}$ состояния $\bar{a}$ и $\bar{b}^{\prime}$ не могут быть эквивалентными.

Вариант 4: $\bar{a} \in C_{2}, \bar{b} \in C_{0}$. В этом случае можно построить $t$-фрагмент, содержащий $\bar{a}$ и поэтому отличный от $F_{0}^{\prime}$; единственность последнего исключает этот вариант. 
Вариант 5: $\bar{a} \in C_{2}, \bar{b} \in C_{1}$. Здесь, как и в варианте 4, можно построить $t$-фрагмент, содержащий $\bar{a}$, то есть отличный от $F_{0}^{\prime}$, и поэтому возникает противоречие с единственностью $t$-фрагмента $F_{0}^{\prime}$.

Вариант 6 , где $\bar{a} \in C_{2}, \bar{b} \in C_{2}$, исключается сразу ввиду того, что $\bar{a} \neq \bar{b}$, а множество $C_{2}$ не имеет различных и эквивалентных принадлежащих ему состояний.

Итак, ситуация, когда $\bar{a}$ - это $p$-состояние, а $\bar{b}$ - это $q$-состояние, разобран полностью. Этим исчерпан и весь случай 3 , так как, поменяв типы состояний $\bar{a}$ и $\bar{b}$, мы получим уже исследованные варианты.

В итоге базис индукции рассмотрен.

Проведем шаг индукции. Пусть $A$ - тупиковый автомат, полученный из $A_{0}$ применением какого-то числа $\varphi$-преобразований, и выполняется еще одно $\varphi$-преобразование.

Тот $s$-фрагмент, принадлежащий автомату $A$, к которому применяется это $\varphi$-преобразование, обозначим через $F$. Пусть $A^{\prime}-$ полученный его применением автомат, и $F^{\prime}=\varphi(F)$.

Как и в случае обоснования базиса индукции, множество состояний автомата $A^{\prime}$ разобьем на подмножества $C_{0}, C_{1}, C_{2}$, руководствуясь теми же правилами.

Из всех ситуаций, рассмотренных для автомата $A_{0}^{\prime}$, при доказательстве тупиковости автомата $A^{\prime}$ нужно остановиться только на тех, в которых использовалась единственность $t$-фрагмента в $A_{0}^{\prime}$. Таких случаев три, и все они состоят в построении $t$-фрагмента, отличного от фрагмента $F^{\prime}$. Обозначим его $\bar{F}$. В каком бы отношении ни находился фрагмент $\bar{F}$ с фрагментом $F^{\prime}$, обратив первый и второй в $s$-фрагменты, мы придем к тупиковому автомату, используя индуктивное предположение. Состояния, одно из которых влечет построение $\bar{F}$, а другое принадлежит ему, не могут быть эквивалентными в этом тупиковом автомате. Шаг индукции проведен. Лемма 2 доказана.

Основной в данном разделе является теорема 3. Предпошлем ей следующее утверждение.

Предложение 16. Множество всех строго входящих в канонический автомат s-фрагментов обладает следующим свойством: какими бы ни были два различных принадлежащчих ему s-фрагмента, либо один из них вложен в другой, либо они не имеют общих принадлежащчих им состояний и общих внешних входов.

Справедливость этого утверждения основана на определении строгого вхождения в автомат $s$-фрагмента.

Теорема 3. Существвет проиедура построения всех минимальных автоматов в главном срезе класса $K$.

Доказательство. Доказательство теоремы 3 осуществляется предъявлением требуемой процедуры. Опишем ее.

Сначала этой процедурой выделяются в каноническом автомате все строго входящие в него $s$-фрагменты с неотрицательной характеристикой. Обозначим $Q$ их множество.

Если $Q$ пусто, то, как легко видеть, канонический автомат является единственным минимальным в $K$, и тогда процедура считает завершенной свою работу, а канонический автомат называем основным. 
В случае, когда $Q$ не пусто, оно разбивается на четыре подмножества:

$Q_{1}=\{s$-фрагменты нулевого ранга с положительной характеристикой $\}$;

$Q_{2}=\{s$-фрагменты ненулевого ранга с положительной характеристикой $\}$;

$Q_{3}=\{s$-фрагменты нулевого ранга с нулевой характеристикой $\}$;

$Q_{4}=\{s$-фрагменты ненулевого ранга с нулевой характеристикой $\}$.

Если $Q_{1} \cup Q_{2}$ не пусто, то процедура формирует множество $\bar{Q}$, состоящее из всех $s$-фрагментов, принадлежащих $Q_{1}$, и из главных представителей фрагментов из $Q_{2}$. Процедурой выполняется в каноническом автомате $\varphi$-преобразование для каждого $s$-фрагмента из $\bar{Q}$. Это возможно на основании предложений 11 и 16, а по лемме 2 процедура трансформирует канонический автомат в тупиковый. По предложению 13, если при выполнении этих $\varphi$-преобразований и возникают новые $s$-фрагменты, то применение к ним $\varphi$-преобразований только увеличивало бы число $q$-состояний в автомате. Отсюда следует, что построенный процедурой автомат действительно является минимальным. Он называется основным минимальным в $K$ автоматом.

Если $Q_{1} \cup Q_{2}$ пусто, то основным является канонический автомат.

В случае, когда в $Q_{2}$ имеются $s$-фрагменты, главные представители которых отличны от них самих, процедурой строятся автоматы, отличные от основного, но тоже являющиеся минимальными в $K$. Для построения любого из них берется множество $s$-фрагментов, состоящее из $s$-фрагментов множества $Q_{1}$ и всех представителей $s$-фрагментов из $Q_{2}$, для которых хотя бы один отличен от главного. Затем процедура применяет $\varphi$-преобразование к каждому $s$-фрагменту из данного множества. Опять-таки осуществимость этого обеспечивается предложениями 11 и 16, а тупиковость построенного автомата - леммой 2. Если при этих $\varphi$-преобразованиях и возникают новые $s$-фрагменты, то их маркеры либо равны маркеру преобразуемого фрагмента, либо меньше его; применение $\varphi$-преобразований к первым не изменило бы числа $q$-состояний в автомате, а применение ко вторым увеличило бы их число. Таким образом, построенный автомат минимален в $K$. Процедурой перебираются все возможные варианты множества $s$-фрагментов, используемого при построении автомата. Так конструируемые автоматы называются первичными. К ним причисляется и основной минимальный автомат.

Далее процедурой рассматривается множество $\overline{\bar{Q}}$, состоящие из всех $s$-фрагментов, принадлежащих $Q_{3}$, и всех представителей $s$-фрагментов, принадлежащих $Q_{4}$. Если $\overline{\bar{Q}}$ не пусто, то для произвольного непустого его подмножества и любого первичного минимального автомата строится новый минимальный автомат посредством выполнения $\varphi$-преобразований для всех фрагментов выбранного подмножества. Каждое из таких $\varphi$-преобразований, меняя структуру автомата, сохраняет в нем число $q$-состояний. Отсюда и следует, что построенный автомат действительно минимален в $K$. Все конструируемые таким образом автоматы называются вторичными.

Все действия процедуры описаны.

Тот факт, что в $K$ нет минимальных автоматов, отличных от первичных и вторичных, построенных процедурой при непустом $Q$, следует из ее описания.

Теорема 3 доказана.

Следствие 2. Любая детерминизациия предложенной процедуры приводит к одному $u$ тому же множеству построенных автоматов.

Основанием этому является то, что применяемые процедурой $\varphi$-преобразования дают результаты, не зависящие от порядка их выполнения. На рис. 12 демонстрируется случай, 
когда канонический автомат не является минимальным; таковым является автомат $A_{2}$, полученный из $A_{1}$ успешным $\varphi$-преобразованием.

\section{Список литературы}

1. Рабин М. О., Скотт Д., Конечные автоматы и проблемы их разрешения. Киберн. сб. (1962) 4, 58-91.

2. Grifliths T. V., The unsolvability of the equivalence problem for $\lambda$-free nondeterministic generalized machines. J. ACM (1968) 15, 409-413.

3. Bird M., The equivalence problem for deterministic two-tape automata. J. Comp. Syst. Sci. (1973) 7, №4, 218-236.

4. Harju T., Karhumaki J., The equivalence problem of multitape finite automata. Theoret. Comp. Sci. (1991) 78, №2, 347-355.

5. Лакхем Д. К., Парк Д. М., О формализованных компьютерных программах. Киберн. сб. (1975) 12, 78-114.

6. Tamm H., On minimality and size reduction of one-tape and multitape finite automata. Univ. Helsinki, Helsinki, 2004.

7. Хачатрян В. Е., Решение обобщенной проблемы минимизации для двухленточных автоматов с одной фиксированной лентой. Докл. РАН (2006) 411, №3, 314-318.

8. Подловченко Р. И. К вопросу об эквивалентных преобразованиях алгоритмов и программ. $M a-$ тем. вопросы киберн. (2000) 9, 25-36.

Статья поступила 25.12.2007. 